\title{
Ultra-subwavelength phase-sensitive Fano-imaging of localized photonic modes
}

\author{
Niccolò Caselli ${ }^{1,2}$, Francesca Intonti ${ }^{1,2}$, Federico La China ${ }^{1,2}$, Francesco Riboli ${ }^{3}$, Annamaria Gerardino ${ }^{4}$, \\ Wei Bao ${ }^{5}$, Alexander Weber Bargioni ${ }^{5}$, Lianhe $\mathrm{Li}^{6}$, Edmund H Linfield ${ }^{6}$, Francesco Pagliano ${ }^{7}$, Andrea Fiore \\ and Massimo Gurioli ${ }^{1,2}$
}

Photonic and plasmonic devices rely on nanoscale control of the local density of optical states (LDOS) in dielectric and metallic environments. The tremendous progress in designing and tailoring the electric LDOS of nano-resonators requires an investigation tool that is able to access the detailed features of the optical localized resonant modes with deep-subwavelength spatial resolution. This scenario has motivated the development of different nanoscale imaging techniques. Here, we prove that a technique involving the combination of scanning near-field optical microscopy with resonant scattering spectroscopy enables imaging the electric LDOS in nano-resonators with outstanding spatial resolution $(\lambda / 19)$ by means of a pure optical method based on light scattering. Using this technique, we investigate the properties of photonic crystal nanocavities, demonstrating that the resonant modes appear as characteristic Fano line shapes, which arise from interference. Therefore, by monitoring the spatial variation of the Fano line shape, we locally measure the phase modulation of the resonant modes without the need of external heterodyne detection. This novel, deep-subwavelength imaging method allows us to access both the intensity and the phase modulation of localized electric fields. Finally, this technique could be implemented on any type of platform, being particularly appealing for those based on non-optically active material, such as silicon, glass, polymers, or metals.

Light: Science \& Applications (2015) 4, e326; doi:10.1038/lsa.2015.99; published online 11 September 2015

Keywords: nanocavity; nanophotonics; near-field; phase retrieval; resonant-scattering

\section{INTRODUCTION}

The development of integrated nanophotonic and nanoplasmonic optical resonators is currently one of the main goals of the nanooptics community. In optical nano-resonators, the electric local density of optical states (LDOS), which assesses the number of available photonic states in a specific spatial region ${ }^{1-4}$, is dominated by a few strongly localized modes that are not spectrally overlapped (Note that for well spectrally resolved modes in nanoresonators the electric LDOS corresponds to the electric field intensity of the modes). The characterization of the electric LDOS at the proper spatial resolution is an essential step toward the realization of high-density photonic integrated circuits and quantum photonic networks ${ }^{5-7}$. For example, photon tunneling in coupled photonic crystal nanocavities (PCNs) is governed by the electric LDOS spatial overlap between neighboring resonators $^{8}$. Similarly, the electric LDOS of a localized mode strongly influences the light-matter interaction of a single quantum emitter (being directly related to the spontaneous emission rate ${ }^{9}$ as well as to the signal amplification ${ }^{10}$ ), and the electric LDOS is crucial for the development of efficient sensors ${ }^{11,12}$. Major advances in tailoring the electric LDOS at the nanoscale have led, on one hand, to a sub-wavelength electric field confinement in metallic nanoantennas to control the directional emission ${ }^{13}$ and, on the other hand, to the achievement of the strong coupling regime, with the requirement of placing the wavelength-matched nano-emitter at the location of the maximum of the PCN electric LDOS at a spatial precision of approximately $\lambda / 15^{14}$.

Currently, many experimental techniques are available for imaging the optical resonant mode features at nanoscale resolution. Scanning near-field optical microscopy (SNOM) photoluminescence is proven to be a powerful technique for mapping the electric LDOS of nanoresonators. For example, in III-V semiconductor photonic platforms, multiple quantum wells or quantum dots are embedded to serve as internal light sources ${ }^{15}$. In systems such as silicon-, glass-, polymer-, or metal-based devices, the modification of the spontaneous emission rate of small emitters (such as fluorescent dyes or nitrogen vacancy centers) can be monitored by placing the light source at the end of a scanning probe $e^{16,17}$. However, photoluminescence techniques require a spectral matching between the emitter and the photonic mode, and especially in case of functionalized probes, they may suffer the phenomena of bleaching and blinking ${ }^{18,19}$.

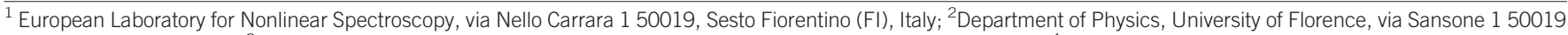

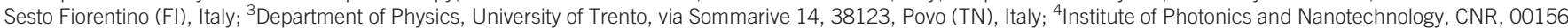

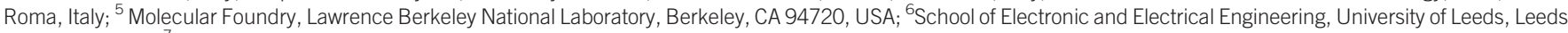
LS2 9JT, UK and ${ }^{7}$ COBRA Research Institute, Eindhoven University of Technology, 5600 MB Eindhoven, The Netherlands 
a

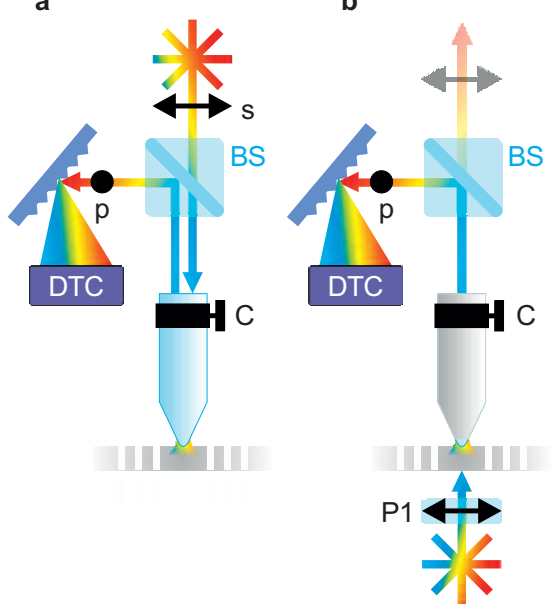

C

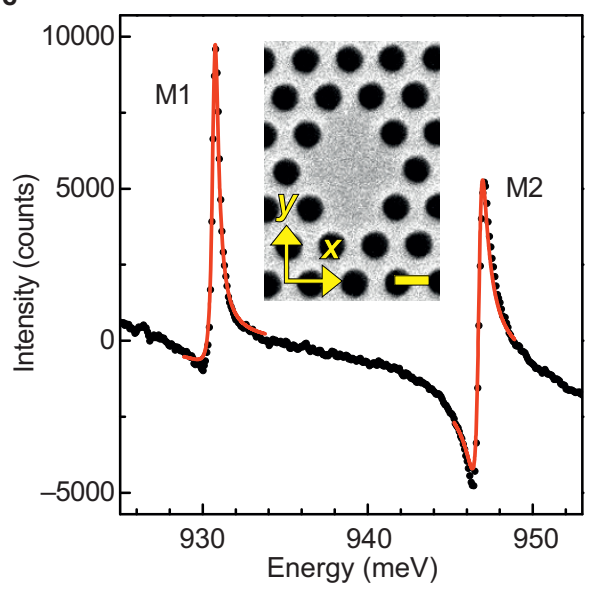

d

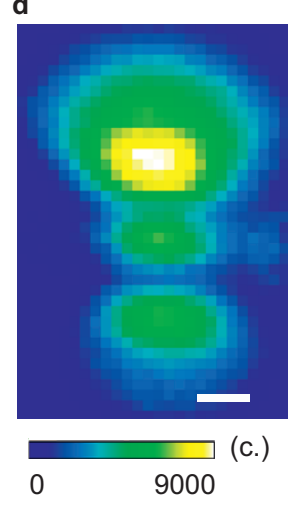

e

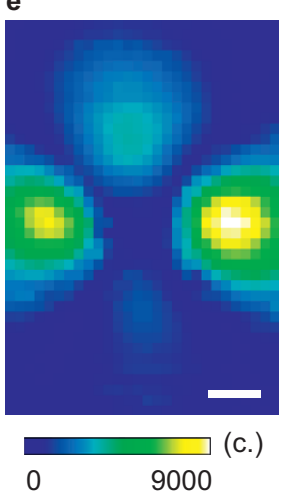

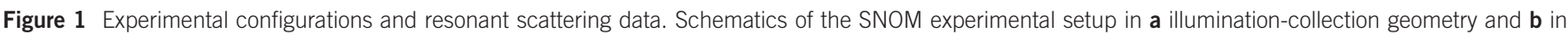

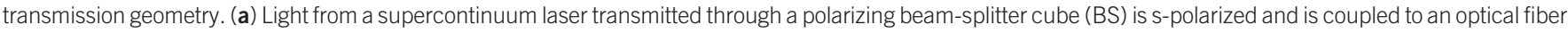

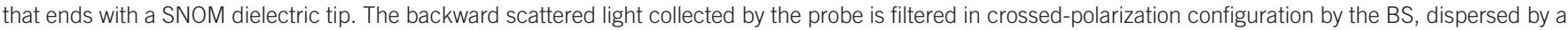

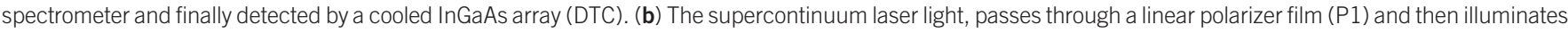

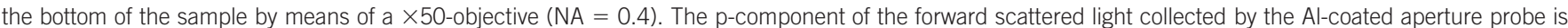

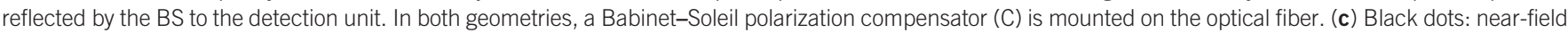
RBS spectrum background-subtracted as a function of energy, collected inside the PCN. Red lines: fitted curves provided by Equation (1) for the M1 and M2 modes.

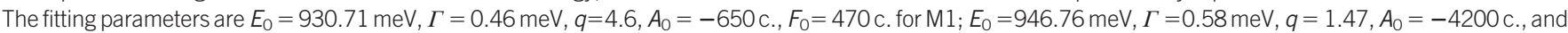

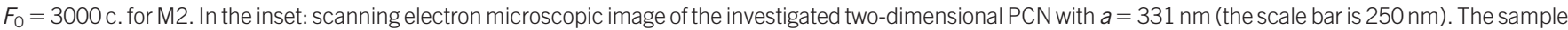

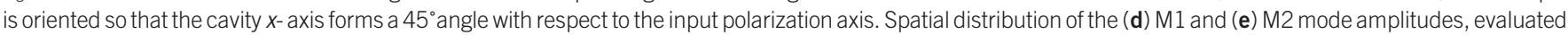
as $F_{0}\left(1+q^{2}\right)$. The scale bars are $250 \mathrm{~nm}$, and the map dimensions are the same as those of the inset in $\mathbf{c}$.

An alternative approach based on cathodoluminescence-enabled deep sub-wavelength (approximately $\lambda / 20$ ) imaging of a two-dimensional $\mathrm{Si}_{3} \mathrm{~N}_{4}$-based PCN in a broad spectral range ${ }^{20}$; however, this technique is limited to the collection of the electric LDOS in dielectric regions, and it is primarily sensitive to the out-of-plane electric field component.

Pure photonic investigation techniques are potentially more powerful due to the direct investigation of a broad spectral range, the possibility to address the distributions of localized modes in air regions or in metal devices, the absence of polarization constraints and the possibility of phase retrieval when used in combination with heterodyne detection in an external interferometer ${ }^{21-23}$. A particularly good example is represented by near-field surface enhanced light scattering performed with apertureless probes, as recently applied to various contexts $^{22,23}$. The high sensitivity of this method is largely due to the high refractive index material probes used to enhance the scattering. However, the disadvantage of this light scattering technique is that the investigation of optical micro- and nano-resonators (e.g., ring resonators, microdisk cavities, and PCNs) excessively perturbs the dielectric environment compared to glass probes $^{24}$, thereby deteriorating the high quality factor $(\mathrm{Q})$ and also detuning/mixing modes localized in photonic molecules or in random cavities ${ }^{8,25}$. Moreover, this technique, as in the case of cathodoluminescence, suffers from being mainly sensitive to the out-of-plane component of the electric LDOS $^{22}$, and this technique is not suited for TE-like localized modes. Therefore, a pure optical method that can be applied on high-Q resonators to retrieve the intensity and the phase of the confined electric field is required.

\section{MATERIALS AND METHODS}

Inspired by far-field resonant scattering experiments, which exhibit Fano-like resonances in high-Q $\mathrm{PCNs}^{26-28}$, we implement a full photonic method based on either the resonant back scattering (RBS) or the resonant forward scattering (RFS) configuration in the near-field regime. We use dielectric tapered SNOM probes to achieve a combined spatial and spectral (hyperspectral) analysis of the electric LDOS of the modes localized in PCNs. In addition, we use aluminumcoated aperture probes and plasmonic probes to retrieve the spatial modulation of the phase of the localized modes. This SNOM Fano imaging technique proves to be phase sensitive because it intrinsically involves the interference between two distinct scattering pathways: (i) light diffused by the surface through a continuum of extended states, which is not coupled to the cavity mode and (ii) light resonantly coupled to the cavity mode and subsequently emitted in free space ${ }^{29-31}$. This interference phenomenon gives rise to Fano resonances, whose line shape is closely related to the phase difference between scattering channels (i) and (ii) described above ${ }^{32}$.

The presented technique can be applied to a large variety of microand nano-resonators that are made of any type of material. The technique does not require any special design or challenging fabrication steps to implement, and the technique is sensitive to different polarization components. Moreover, the use of white light generated by a supercontinuum laser ensures the hyperspectral imaging in the full optical range of interest.

We use a commercial SNOM (TwinSnom, Omicron GmbH, Taunusstein, Germany) in two different configurations: the illumination-collection geometry (see Figure 1a) and the transmission geometry (see Figure 1b) to detect the RBS and the RFS, respectively. In the illumination/collection geometry, we perform crossed-polarization detection to overcome the huge reflection signal, which has the same polarization as that of the incident light. In fact, by only balancing the diffused and the resonant signals we clearly observe Fano line shapes. Therefore, the implementation of SNOM resonant scattering requires polarization control in both the input and the detection 
channel paths, which can share the same near-field probe. In particular, light coming from a supercontinuum laser (ranging in a photon energy range from $0.8 \mathrm{eV}$ to $1.0 \mathrm{eV}$ ) is linearly polarized via transmission through a polarizing beam splitter cube, whose extinction ratio is $2 \times 10^{4}$, and then it is coupled to a dielectric probe to illuminate the sample in the near-field. A Babinet-Soleil compensator is mounted on the optical fiber to control the light polarization at the end of the tip, leading to a linear polarization of the light (in the $940 \pm 20 \mathrm{meV}$ spectrum energy range) incident onto the sample at $45^{\circ}$ with respect to the $x$ axis of the PCN (as defined in the inset of Figure 1c). This illumination condition ensures the best signal-to-noise ratio in the crossed-polarization configuration. The backward scattered light, which is collected by the same probe, is filtered in the crossed-polarization configuration by the polarizing beam-splitter cube, dispersed by a spectrometer, and finally detected by a cooled InGaAs array; the resulting spectral resolution is $0.08 \mathrm{meV}$.

In the transmission geometry, to retrieve separately the $x$ - and $y$-phase spatial modulation of the localized modes, we illuminate the sample alternatively with light polarized parallel to the $x$ - or $y$-axis of the PCN, thus relaxing the crossed-polarization condition in the detection scheme. Although this setup does not guarantee the best signal-to-noise ratio, when we use an Al-coated aperture near-field probe (LovaLite, Besancon, France), we succeed in balancing the scattering channels (i) and (ii) for each independent polarization. In fact, different from the dielectric probes, metalized aperture probes collect light only from the small aperture placed a few nanometers on top of the sample, thereby spatially filtering out the non-resonant signal in a very efficient manner. In particular, the supercontinuum laser light used is linearly polarized by a Glan-Thompson polarizing prism (P1), with an extinction ratio of approximately $3 \times 10^{3}$. The linearly polarized light is focused on the sample surface by means of a $50 \times$-objective $(\mathrm{NA}=0.4)$ in the far-field regime. The illumination spot diameter size is approximately $2 \mu \mathrm{m}$ to ensure a homogeneous excitation on the entire PCN plane with fixed relative phase. Next, the transmitted light is collected by the Al-coated probe, and the polarizing beam-splitter filters out the polarization components that are orthogonal to the incident light. Finally, the signal is analyzed by the same detection scheme used in the RBS setup.

In both geometries, the following apply: (i) the Babinet-Soleil compensator is mounted on the optical fiber to provide a linear polarization response of the system fiber plus tip with an extinction ratio of 120; (ii) the tip remains fixed while the sample is raster scanned at a constant tip-sample distance; and (iii) the spectra are collected at every tip position on the sample surface.

In this study, the samples under investigation are two-dimensional photonic crystal cavities fabricated on a 320-nm thick GaAs suspended membrane to ensure confinement in the vertical direction due to total internal reflection. The photonic crystals consist of a twodimensional triangular lattice of air holes with a lattice constant of either $a=321 \mathrm{~nm}$ or $a=331 \mathrm{~nm}$ and a filling fraction of $35 \%{ }^{33}$. The PCNs are formed by four missing holes organized in a diamond-like geometry $^{34}$, as shown in the scanning electron microscopic (SEM) image shown in the inset of Figure 1c.

\section{RESULTS AND DISCUSSION}

A typical RBS spectrum of the investigated PCN is shown in Figure 1c. The spectrum displays two resonances corresponding to the two lower energy modes (M1 and M2) and includes both positive and negative values because the experimental background signal (which is spatially averaged in a region at approximately $2 \mu \mathrm{m}$ away from the cavity) has been subtracted from the raw data. Both resonances exhibit a dispersive-like line shape, whose intensity $F$ as a function of energy is well reproduced (see Figure $2 \mathrm{a}$ ) by the Fano formula ${ }^{26,29}$ :

$$
F(E)=A_{0}+F_{0} \frac{\left[q+2\left(E-E_{0}\right) / \Gamma\right]^{2}}{1+\left[2\left(E-E_{0}\right) / \Gamma\right]^{2}}
$$

where $A_{0}$ and $F_{0}$ are amplitude factors and $E_{0}$ and $\Gamma$ are the energy and the linewidth of the PCN mode, respectively, while the dimensionless parameter $q$ accounts for the line-shape asymmetry. To map the RBS amplitude for both M1 and M2, we report, for each mode as a function of position, the value $F_{0}\left(1+q^{2}\right)$ provided by the fit, which corresponds to the difference in the counts between the maximum and the minimum of the Fano line shape, as shown in Figure 1d and 1e. These maps reproduce the main features of the electric LDOS at the energy of the resonant modes obtained by finite difference time domain (FDTD) calculations, as shown in Figure $2 \mathrm{c}$ and $2 \mathrm{~g}$. However, these figures show a limited spatial resolution (approximately 250 $\mathrm{nm}$ ) because the amplitude map is directly proportional to the light collected by the dielectric uncoated near-field probe, whose resolution is limited to the few hundred nanometers of the collecting area. In fact, tapered dielectric probes collect signal not only from the tip apex but also from the sidewalls, resulting in a collection area of approximately $250 \mathrm{~nm}$.

An improved spatial resolution, down to $\sim \lambda / 19$, is achievable from the same set of data by making best use of the tip local perturbation on the PCN dielectric environment. The slight perturbation induced by the apex of the near-field tip gently shifts the resonant modes toward lower energies in proportion to the strength of the unperturbed electric field intensity at the position of the tip apex, as extensively demonstrated in Refs. 24 and 34. This phenomenon is underlined by the spectra shown in Figure $2 \mathrm{a}$, which are collected at two different positions of the PCN surface, as highlighted by the green and the red circles of Figure $2 \mathrm{~b}$.The tip perturbation only slightly alters the Q-factor of the bare resonator (evaluated as $\left.E_{0} / \Gamma\right)$. In fact, using a far-field setup equivalent to the one of Ref. 26, we obtain Q $=2650$ for M1. In the SNOM measurement, we observed a Q reduction in the range of $5 \%-20 \%$ of the original value, i.e., the perturbation is strong enough to provide a detectable spectral shift but still not so large to alter the localized mode spatial distribution.

Figure $2 \mathrm{~b}$ and $2 \mathrm{f}$ shows the maps of the tip induced spectral shift for M1 and M2, respectively, as obtained by reporting for each mode $E_{0}$ as a function of position. The results are in excellent agreement with the FDTD calculations shown in Figure $2 \mathrm{c}$ and $2 \mathrm{~g}$. To estimate the spatial resolution of the Fano imaging technique, because the smallest spatial feature shown in Figure $2 \mathrm{c}-2 \mathrm{~g}$ is not delta-like, we compare the experimental map of Figure $2 b$ to the theoretical maps obtained by the convolution between the FDTD-calculated distribution and twodimensional Gaussian point spread functions, which are characterized by different full width at half maximum (FWHM) values. In particular, the experimental spectral shift data along the profiles shown in Figure $2 \mathrm{c}$ as dotted lines are well reproduced by the profiles obtained on the FDTD map convoluted with a Gaussian function of $70 \mathrm{~nm}$ FWHM, as shown in Figure 2d and 2e.

The estimation is provided by the method of maximum likelihood, thus minimizing the residual sum of squares weighted with the experimental uncertainties. The fit quality is measured by the value of the reduced $\chi^{2}$ parameter. We thus obtain as the best fit the point-spread-function of $70 \mathrm{~nm}$ FWHM with a reduced $\chi^{2}$ equal to 1.1 and 1.2 for the data shown in Figure $2 \mathrm{~d}$ and $2 \mathrm{e}$, respectively. 
a

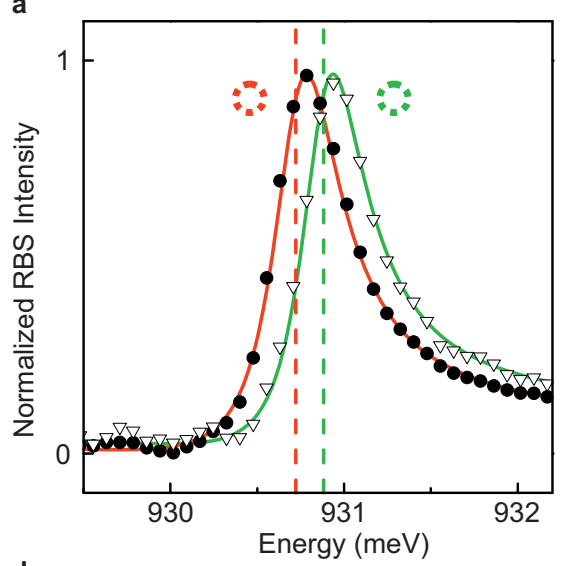

d

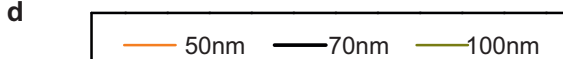

b

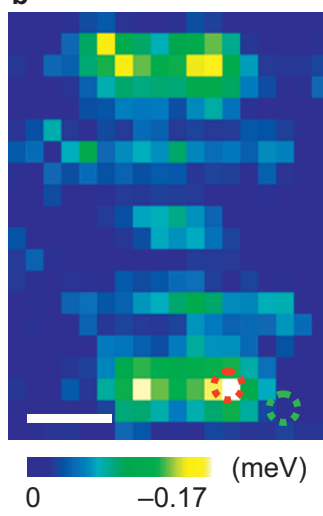

c

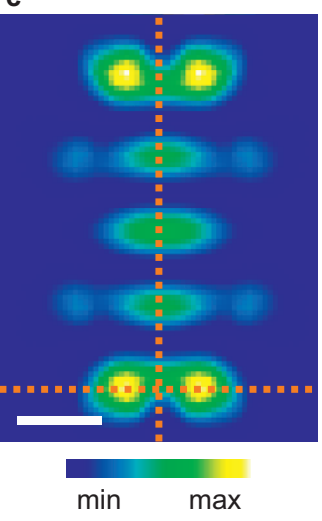

e

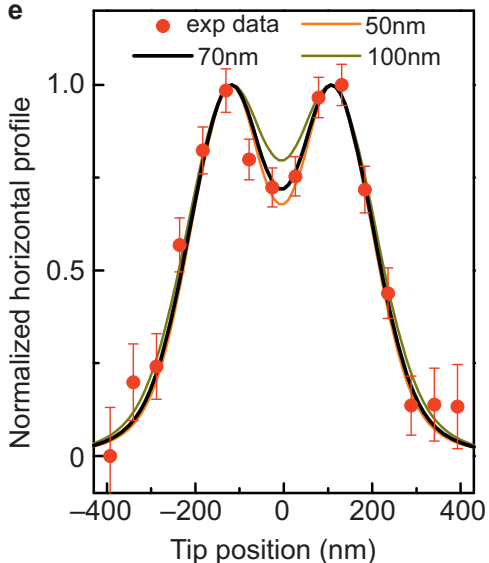

f

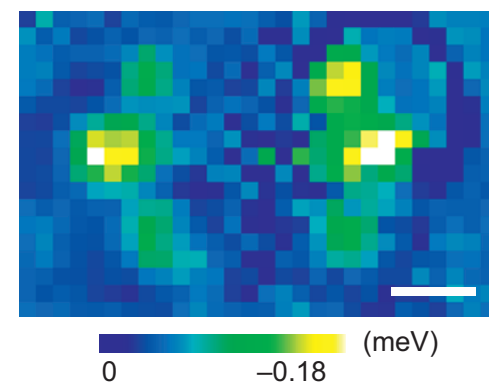

g

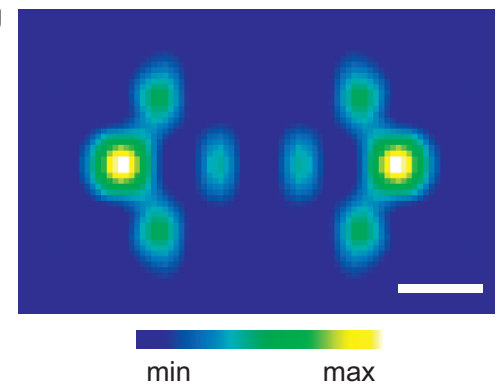

Figure 2 Ultra-subwavelength Fano LDOS imaging. (a) Normalized RBS spectra of the mode M1 of the PCN with $a=331 \mathrm{~nm}$, collected at two different tip positions, as highlighted by red and green circles in $\mathbf{b}$, and here denoted as dots and triangles, respectively. The solid curves provide the fit results using Equation (1), whose resonant energies $E_{0}$ are highlighted by the red and green vertical dashed lines, respectively. The fitted parameters are $E_{0}=930.72 \mathrm{meV}, \Gamma=0.47 \mathrm{meV}, q=4.4$, $A_{0}=0.010$, and $F_{0}=0.049$ for the red curve, and $E_{0}=930.88 \mathrm{meV}, \Gamma=0.45 \mathrm{meV}, q=3.9, A_{0}=0.035$, and $F_{0}=0.049$ for the green curve. The red spectrum, which shows the largest tip perturbation, was collected in the proximity of the maximum of the M1 electric field intensity, corresponding to the red circle in $\mathbf{b}$. The green spectrum was collected at the green circle position in $\mathbf{b}$, where the PCN experiences a negligible tip perturbation. (b) M1 mode map of the tip induced spectral shift, by plotting $E_{0}$ as a function of tip position. (c) Map of the electric LDOS intensity calculated using the FDTD method at the energy of the M1 mode. The dotted orange lines in $\mathbf{c}$ indicate the positions where the profiles shown in $\mathbf{d}$ and $\mathbf{e}$ are considered. The letters $\mathrm{A}, \mathrm{B}$ and $\mathrm{C}$ indicate the lobes where the spectra of Figure $3 \mathrm{a}$ are collected. (d) Comparison between the vertical profiles obtained from the normalized map of the experimental data (red dots) and from the normalized convolution between the calculated electric LDOS and a two-dimensional Gaussian point-spread function of FWHM of $70 \mathrm{~nm}$. The error bars are provided by the Fano function fit of each spectrum acquired. (e) Same as d, for the horizontal profile. (f) Experimental spectral shift map and (g) FDTD electric LDOS map evaluated at the energy of the M2 mode. The scale bar in all the maps is $250 \mathrm{~nm}$.

In addition, we are able to provide an estimation of the effective tip apex size (which is responsible for the dielectric perturbation) using the relations found in Ref. 24 that link this quantity to the measured maximum spectral shift and parameters evaluated by the FDTD calculations. These parameters for the M1 mode are the mode decay length in free space beyond the slab $d=40 \mathrm{~nm}$ and the mode volume $\mathrm{V}_{\text {cav }}=\frac{\int \varepsilon(\vec{r})\left|\vec{E}_{0}(\vec{r})\right|^{2} d \vec{r}}{\max \left[\varepsilon(\vec{r})\left|\vec{E}_{0}(\vec{r})\right|^{2}\right]}=0.033 \mu \mathrm{m}^{3}$, where $\varepsilon(\vec{r})$ is the PCN dielectric constant (equal to 12.14 in GaAs and equal to 1 in air) and $\vec{E}_{0}(\vec{r})$ is the FDTD-calculated electric field without the presence of the tip. With these parameters, the tip electric polarizability is estimated as $\alpha=1.1 \times 10^{-21} \mathrm{~m}^{3} \varepsilon_{0}$. Next, defining the tip refractive index as $n_{\mathrm{T}}=1.5$, we obtain the effective tip apex radius: $R=\sqrt{\frac{\alpha\left(n_{\mathrm{T}}^{2}+2\right)}{3 \pi d \varepsilon_{0}\left(n_{\mathrm{T}}^{2}-1\right)}} \cong 100 \mathrm{~nm}$. The effective tip apex dimension $R$ obtained with the simple dielectric probe model of Ref. 24 agrees with the probe dimensions extracted 
a

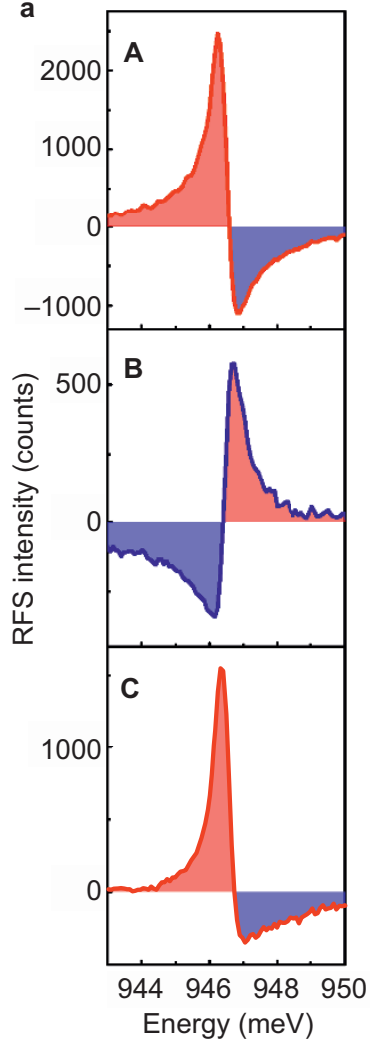

b

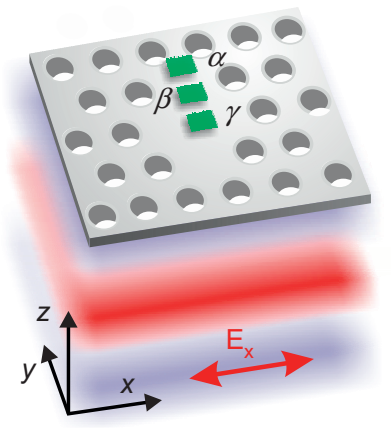

d

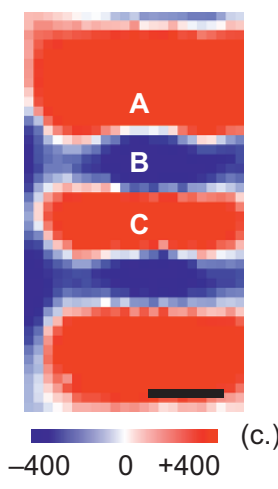

f

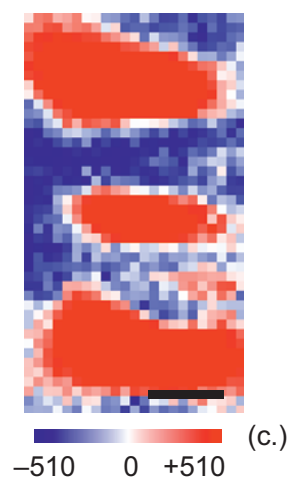

c

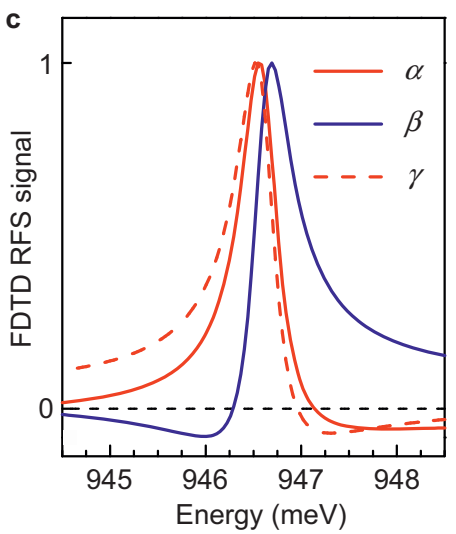

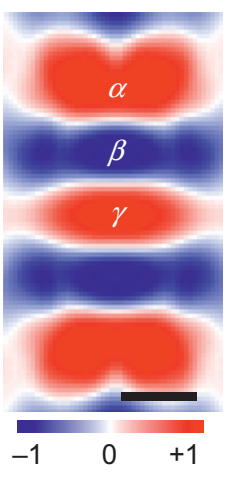

(c.)

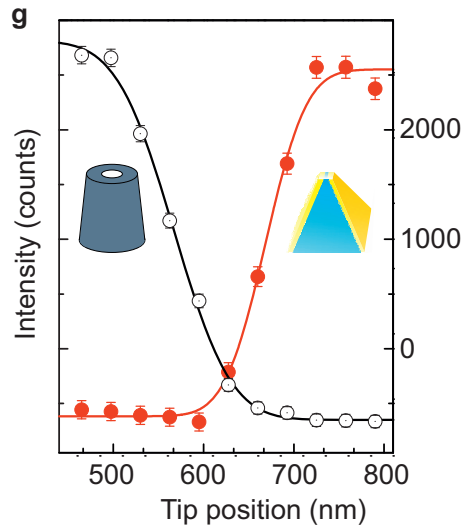

Figure 3 Resonant scattering fundamental mode phase imaging. (a) RFS spectra of M1 collected by the Al-coated probe at three positions on the PCN, corresponding to the center of the lobes A, B and C in $\mathbf{d}$ and in Figure 2c. The red (blue) areas highlight the constructive (destructive) interference. (b) Schematic of the FDTD calculations used to model the RFS imaging process. The PCN is excited from the bottom by a linearly polarized plane wave, and three detectors (green squares) centered on the lobes, indicate by $\alpha, \beta$, and $\gamma$ in $\mathbf{e}$, are placed $10 \mathrm{~nm}$ above the sample surface. (c) Calculated RFS spectra collected by the three detectors in $\mathbf{b}$; each spectrum is background subtracted and divided by its own maximum. (d) Map of the RFS intensity evaluated at 945.90 meV. All the spectra recorded in the red (blue) regions have line shapes similar to the A (B) spectrum in a. (e) Real part of the $x$-component of the M1 electric field calculated using the FDTD method and normalized in $[-1 ;+1]$. The phase difference between the lobes $\alpha$ and $\beta$ is equal to $\pi$. (f) RBS intensity map of M1 collected using the Campanile tip, evaluated at the proper energy. (g) Red dots and black circle are the intensity data collected by Campanile and Al-coated probe, respectively, as a function of position. The schematics of the probes are shown in the inset. Data are acquired along the vertical profiles between adjacent lobes in $\mathbf{d}$ and $\mathbf{f}$ and are shifted along the position axis. The error bars are provided as the square root of the intensity counts. Red and black curves are the error functions that best reproduce the observed data.

from the SEM images of the dielectric tips and is of the same order of magnitude with respect to the Fano electric LDOS imaging spatial resolution experimentally evaluated in Figure $2 \mathrm{~d}$ and 2e. Therefore, the combination of SNOM and RBS spectroscopy allows us to obtain high-fidelity electric LDOS mode imaging at an ultra-subwavelength spatial resolution of $70 \mathrm{~nm}(\lambda / 19)$, which is comparable to the best result obtained to date on $\mathrm{PCNs}^{20}$. Note that the Fano imaging does not require any active emitter and it is not limited to detection of a single spatial component of the electric LDOS as in Refs. 20 and 22. Therefore, the Fano imaging technique can be potentially applied to nanostructures that confine light in silicon-based materials as well as glass, polymer, or metallic materials. For example, in the case of plasmonic nanocavities, the proposed near-field all-optical method could also allow for imaging of plasmonic dark modes, which are not addressable by far-field measurements ${ }^{35,36}$.

Moreover, different from photoluminescence experiments, the near-field resonant scattering maps also contain additional information regarding the spatial phase modulation of the modes. In fact, resonant scattering is a coherent signal, and the shape of the Fano resonance is determined by the interference between the non-resonant light diffused with a constant phase over the whole PCN and the resonant signal, which has a well-defined phase spatial dependence (with variations of $\pi$ between adjacent intensity lobes). In our imaging setup, by examining the changes of the Fano line shape as a function of tip position, we obtain an equivalent integrated interferometer to locally probe the relative phase distribution of the PCN localized modes. To demonstrate this capability, we implemented the experimental setup in transmission geometry, as shown in Figure 1b. Figure 3 a shows three RFS spectra relative to the $x$-polarized mode $\mathrm{M} 1$ and collected using an Al-coated aperture tip at three different positions, corresponding to the center of the lobes denoted by A, B, and $\mathrm{C}$ in Figure $2 \mathrm{c}$. The incident light is $x$-polarized. The backgroundsubtracted spectra have positive or negative values, depending on the interference condition between the two scattering channels. In particular, the spectra collected in $\mathrm{A}$ and $\mathrm{C}$ show constructive interference for lower energies, while for higher energies, the interference is destructive. In contrast, the spectrum collected in B has an opposite line shape to those of $\mathrm{A}$ and $\mathrm{C}$, and this inversion is a clear indication that the resonant mode $\mathrm{M} 1$ in $\mathrm{B}$ is characterized by a $\pi$ phase shift with respect to the $\mathrm{A}$ and $\mathrm{C}$ regions. The recovery of the $\mathrm{A}$ line shape in the $\mathrm{C}$ position indicates that the photonic mode has the same phase in both A and C. We emphasize that we only need to consider the lineshape inversions of the spectrum as a function of the tip position and are not interested in the particular line shape because the Fano line 

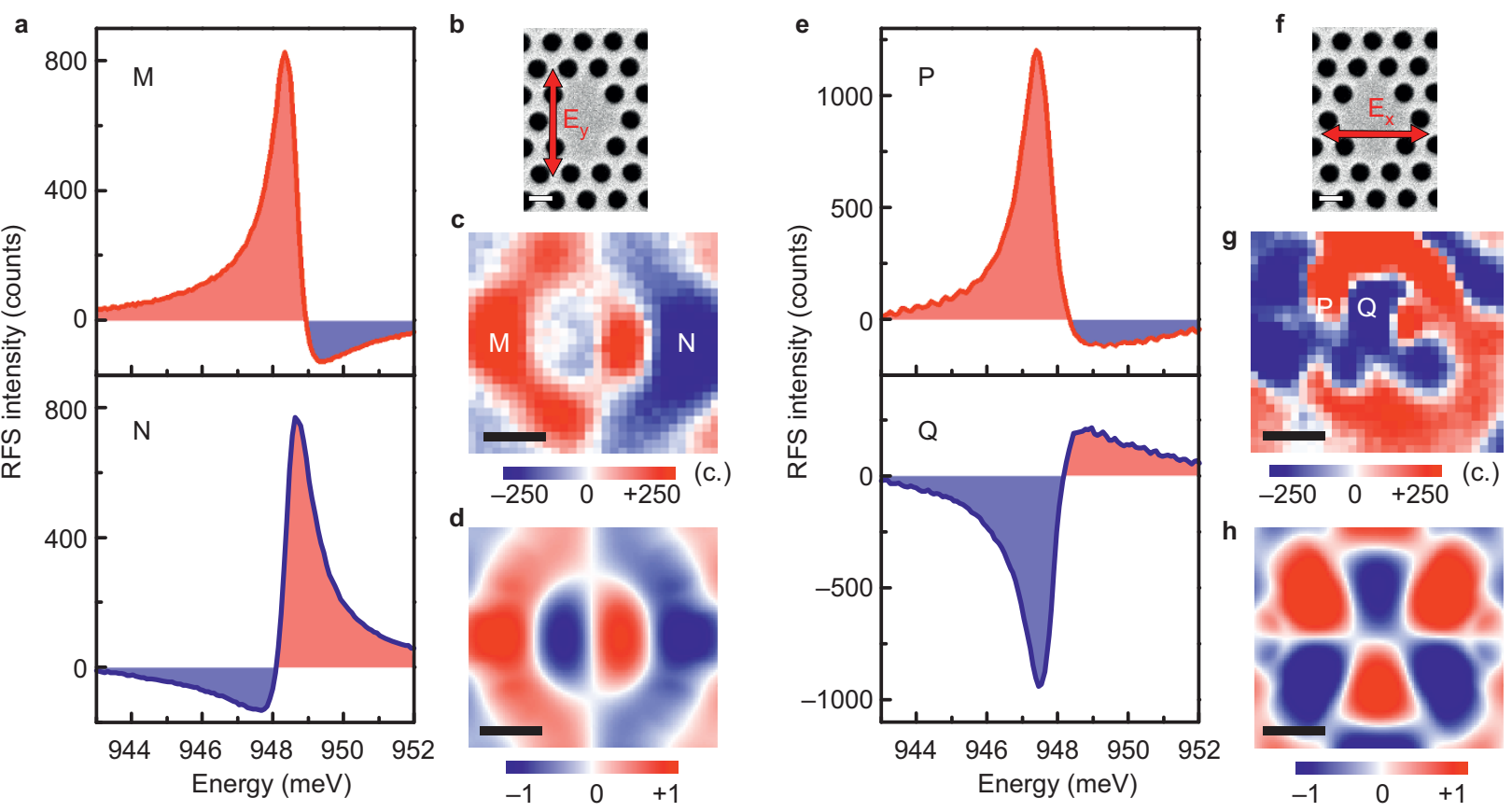

Figure 4 Resonant scattering excited mode phase imaging. (a) RFS spectra of the PCN excited mode M2, collected by the Al-coated probe in the positions M and N shown in $\mathbf{c}$. As highlighted by the schematic in $\mathbf{b}$, the incoming light incident on the sample has a linear polarization along the $y$ axis. (c) Spatial distribution of the $y$ component RFS intensity evaluated at $947.20 \mathrm{meV}$. (d) Real part of the $y$-component of the M2 electric field calculated using the FDTD method, normalized in $[-1 ;+1]$. (e) RFS spectra of M2 collected by the Al-coated probe in the positions P and Q shown in $\mathbf{g}$ for the case of $x$-polarized incident light, as shown in (f). (g) Spatial distribution of the $x$-component RFS intensity evaluated at $947.20 \mathrm{meV}$. (h) Real part of the $x$-component of the M2 electric field calculated using the FDTD method, normalized in $[-1 ;+1]$. The scale bar in all the maps is $250 \mathrm{~nm}$.

shape also depends on the daily setup alignment (which modifies the non-resonant signal) and on the electric LDOS intensity. In fact, we tested the method's reproducibility by investigating many distinct PCNs that are nominally identical but affected by fabrication induced disorder or with different lattice parameters. Even if the absolute Fano line shape is not exactly reproducible, we always observe the same spatial modulation of the resonance line shape as a function of position. To confirm the ability to retrieve the mode phase modulation through Fano line shape changes, we performed three-dimensional FDTD calculations using a commercially available solver package (Crystal Wave 4.9, Photon Design, Oxford, United Kingdom) to schematize the RFS experimental setup, as shown in Figure $3 \mathrm{~b}$. A linearly $x$-polarized plane wave impinges the bare nanocavity, and the signal is collected by three detectors placed above the sample surface at the positions indicated by $\alpha, \beta$, and $\gamma$ in Figure $3 \mathrm{e}$. Figure $3 \mathrm{c}$ shows that the normalized spectra calculated in $\alpha$ and $\gamma$ are characterized by a similar line shape, which differs only because the electric LDOS has distinct values in the two positions (i.e., the ratio between resonant and non-resonant scattering is slightly changed). The spectrum collected by the detector at $\beta$, where the phase is reversed by $\pi$ with respect to the $\alpha$ and $\gamma$ positions, shows an inversion along the energy axis without any effective change of the resonant energy $E_{0}$ compared to the spectra calculated in the other positions. These calculations provide the interference reversal evidence in the spectral range of the resonant mode as a function of position. For imaging purposes, the simplest approach to highlight the spatial modification of the resonance line shape is by plotting the RFS intensity map at a proper energy, as shown in Figure 3d. The spatial sign alternation along the $y$-direction is in good agreement with the phase modulation of the calculated real part of the $x$-component of the electric field shown in Figure $3 \mathrm{e}$, which is the dominant component of the M1 mode. We estimate the spatial resolution of the phase-sensitive Fano imaging by considering a vertical line profile between two adjacent lobes ( $A$ and $B$ in Figure 3d) denoted as the black circle in Figure $3 \mathrm{~g}$. The profile shows an inversion between constructive and destructive interference, which corresponds to an inversion of the Fano line shapes. Therefore, by assuming that the phase is a step-like function and that the experimental point spread function is a Gaussian, we fit the experimental data with an error function, as denoted by the black curve in Figure 3g. By considering the Gaussian FWHM of our experimental resolution, we find that the RFS setup equipped with the Al-coated aperture probe allows for mapping the phase modulations with a spatial resolution of $115 \mathrm{~nm}$. This resolution is limited by the probe aperture, which is nominally approximately $200 \mathrm{~nm}$, and can be improved by using smaller apertures, as demonstrated in the following discussion.

To extend the generality of the method, we tested the Fano phase imaging by employing a plasmonic near-field probe known as a Campanile tip $^{37}$. The inset of Figure $3 \mathrm{~g}$ reports the schematic of the nanoscale aperture plasmonic probe, which has already been tested in SNOM photoluminescence configurations. This probe is characterized by a larger throughput and by a better resolution with respect to standard metal coated tips. Therefore, this probe also allows for examination of the phase imaging in the RBS setup. In fact, with the Campanile probe in the RBS configuration, we obtained spatial line shape inversions that result in the intensity map shown in Figure 3f, which agrees well with the map collected by the Al-coated probe of Figure $3 \mathrm{~d}$. The resolution is estimated using an error function fit of a vertical profile cut of Figure 3f. The data and the relative fit are shown in Figure $3 \mathrm{~g}$ (red dots and red line), corresponding to a spatial resolution of $85 \mathrm{~nm}$. We conclude that the use of resonant scattering is a 
robust method to perform phase retrieval, which does not depend on the details of the probe used.

Thus far, we investigated the M1 mode, which is nearly linearly polarized. However, we can also address the phase modulation of the PCN excited mode M2, which has an elliptical polarization, where both the $x$ - and $y$-components are present ${ }^{38}$. Because the phase distributions of the two perpendicular components are quite different, as shown in Figure $4 \mathrm{~d}$ and $4 \mathrm{~h}$, we must experimentally separate them. Therefore, we illuminate the sample alternatively with $x$ - or $y$-polarized light.

The spectra in Figure 4a and 4e show that the signal-to-noise ratio is still quite high, which allows us to image the phase modulation distribution of the $x$ - and $y$-polarization components of M2 by mapping the mode intensity, as shown in Figure $4 \mathrm{c}$ and $4 \mathrm{~g}$, respectively. As inferred by the comparison between the maps in Figure $4 \mathrm{c}$ and $4 \mathrm{~g}$ and the real part of the $x$ - and $y$-components of the electric field calculated using the FDTD method, shown in Figure 4d and 4h, respectively, the image fidelity of the experimental phase modulation is good. In particular, the map fidelity is higher for the dominant polarization component (parallel to the $y$ axis) with respect to the weaker orthogonal polarization component (parallel to the $x$ axis). The lower fidelity of the $x$ polarization is tentatively attributed to the concomitance of several experimental issues related to the challenge of detecting the RFS in the cross-polarized channel with respect to the dominant mode component. Nevertheless, the $x$-polarization map qualitatively reflects the calculated map, in particular, the mode symmetry and the expected number of phase inversions are observed. These results prove that the described experimental configuration for Fano phase-modulation imaging can be applied to localized modes that exhibit arbitrary polarizations.

The good fidelity with which the maps of the RFS intensity reproduce the distributions of the sign inversions of real part of the electric fields demonstrates the actual phase-modulation imaging of the localized photonic modes. The local changes of the Fano line shape as a function of position provide direct access to the spatial modulation of the localized mode phase by means of an integrated interferometer.

\section{CONCLUSIONS}

In conclusion, we demonstrated a technique of ultra-subwavelength Fano-imaging of the electric LDOS and observed phase modulation of the localized modes in a two-dimensional PCN using a pure optical effect that bridges together resonant light scattering and near-field spectroscopy. This proposed technique has the considerable advantages of being applicable to resonators based on any type of material and of providing imaging over a wide spectral range. Moreover, this proposed technique avoids the bleaching and the strong tip-induced perturbation that degrade the high Q-factors of nano-resonators. In addition, we directly probed the relative phase modulation of the localized mode without the use of an external interferometer by exploiting the nature of the Fano resonances. Our work demonstrates that the near-field Fano imaging technique is a very powerful technique to address the fundamental features of localized modes in optical nano-resonators. This result provides new strategies to investigate at deep-subwavelength spatial resolution the electric LDOS and the phase distribution of modes localized in a variety of nanophotonic and nanoplasmonic resonators, which can be based on materials without optical emission.

\section{ACKNOWLEDGEMENTS}

This work was supported by the FET project FP7 618025 CARTOON and is part of the research program of the Foundation for Fundamental Research on
Matter (FOM), which is financially supported by the Netherlands Organization for Scientific Research (NWO).

1 Busch K, John S. Photonic band gap formation in certain self-organizing systems. Phys Rev E 1998; 58: 3896.

2 McPhedran RC, Botten LC, McOrist J, Asatryan AA, de Sterke CM et al. Density of states functions for photonic crystals. Phys Rev E 2004; 69: 016609.

3 des Francs GC, Girard C, Weeber J-C, Dereux A. Relationship between scanning nearfield optical images and local density of optical states. Chem Phys Lett 2001; 345: 512-516.

4 Joulain K, Carminati R, Mulet J-P, Greffet J-J. Definition and measurement of the local density of electromagnetic states close to an interface. Phys Rev B 2003; 68: 245405.

5 Hausmann BJM, Bulu IB, Deotare PB, McCutcheon M, Venkataraman V et al. Integrated high-quality factor optical resonators in diamond. Nano Lett 2013; 13: 1898-1902.

6 Ritter S, Nolleke C, Hahn C, Reiserer A, Neuzner A et al. An elementary quantum network of single atoms in optical cavities. Nature 2012; 484: 195-200.

7 Kuramochi E, Nozaki K, Shinya A, Takeda K, Sato T et al. Large-scale integration of wavelength-addressable all-optical memories on a photonic crystal chip. Nature Photonics 2014; 8: 474

8 Caselli N, Intonti F, Bianchi C, Riboli F, Vignolini S et al. Post-fabrication control of evanescent tunnelling in photonic crystal molecules. Appl Phys Lett 2012; 101: 211108.

9 Altug H, Englund D, Vučković J. Ultrafast photonic crystal nanocavity laser. Nat Phys 2006; 2: 484-488.

10 Nie S, Emory SR. Probing single molecules and single nanoparticles by surfaceenhanced Raman scattering. Science 1997; 275: 1102.

11 Posani KT, Tripathi V, Annamalai S, Weisse-Bernstein NR, Krishnaa S et al. Nanoscale quantum dot infrared sensors with photonic crystal cavity. Appl Phys Lett 2006; 88: 151104.

12 Anker JN, Hall WP, Lyandres O, Shah NC, Zhao J et al. Biosensing with plasmonic nanosensors. Nat Mater 2008; 7: 442-453.

13 Taminiau TH, Stefani FD, Segerink FB, van Hulst NF. Optical antennas direct singlemolecule emission. Nat Photonics 2008; 2: 234-237.

14 Hennessy K, Badolato A, Winger M, Gerace D, Atatüre M et al. Quantum nature of a strongly coupled single quantum dot-cavity system. Nature 2007; 445: 896-899.

15 Louvion N, Gerard D, Mouette J, de Fornel F, Seassal C et al. Local observation and spectroscopy of optical modes in an active photonic-crystal microcavity. Phys Rev Lett 2005; 94: 113907.

16 Krachmalnicoff V, Cao D, Caze A, Castanie E, Pierrat R et al. Towards a ful characterization of a plasmonic nanostructure with a fluorescent near-field probe. Opt Express 2013; 21: 11536.

17 Frimmer M, Chen Y, Koenderink AF. Scanning emitter lifetime imaging microscopy for spontaneous emission control. Phys Rev Lett 2011; 107: 123602.

18 Nirmal M, Dabbousi BO, Bawendi MG, Macklin JJ, Trautman JK et al. Fluorescence intermittency in single cadmium selenide nanocrystals. Nature 1996; 383: 802804.

19 van Sark WGJHM, Frederix PLTM, Bol AA, Gerritsen HC, Meijerink A. Blueing, bleaching, and blinking of single CdSe/ZnS quantum dots. Chem Phys Chem 2002; 3: 871-879

20 Sapienza R, Coenen T, Renger J, Kuttge M, van Hulst NF et al. Deep-subwavelength imaging of the modal dispersion of light. Nat Mater 2012; 11: 781-787.

21 Burresi M, Engelen RJP, Opheij A, van Oosten D, Mori D et al. Observation of polarization singularities at the nanoscale. Phys Rev Lett 2009; 102: 033902.

22 Schnell M, García-Etxarri A, Huber AJ, Crozier K, Aizpurua J et al. Controlling the nearfield oscillations of loaded plasmonic nanoantennas. Nat Photonics 2009; 3: 287291.

23 Chen JN, Badioli M, Alonso-González P, Thongrattanasiri S, Huth F et al. Optical nanoimaging of gate-tunable graphene plasmons. Nature 2012; 487: 77-81.

24 Koenderink AF, Kafesaki M, Buchler BC, Sandoghdar V. Controlling the resonance of a photonic crystal microcavity by a near-field probe. Phys Rev Lett 2005; 95 : 153904.

25 Riboli F, Caselli N, Vignolini S, Intonti F, Vynck K et al. Engineering of light confinement in strongly scattering disordered media, Nat Mater 2014; 13: 720-725.

26 Galli M, Portalupi SL, Belotti M, Andreani LC, O'Faolain L et al. Light scattering and Fano resonances in high-Q photonic crystal nanocavities. Appl Phys Lett 2009; 94: 071101.

27 Haddadi S, Le Gratiet L, Sagnes I, Raineri F, Bazin A et al. High quality beaming and efficient free-space coupling in L3 photonic crystal active nanocavities. Opt Express 2012; 20: 18876-18886.

28 Englund D, Faraon A, Fushman I, Stoltz N, Petroff P et al. Controling cavity reflectivity with a single quantum dot. Nature 2007; 450: 857-861.

29 Fano U. Effects of configuration interaction on intensities and phase shifts. Phys Rev 1961; 124: 1866.

30 Miroshnichenko AE, Flach S, Kivshar YS. Fano resonances in nanoscale structures. Rev Mod Phys 2010; 82: 2257. 
31 Luk'yanchuk B, Zheludev NI, Maier SA, Halas NJ, Nordlander P et al. The Fano resonance in plasmonic nanostructures and metamaterials. Nat Mater 2010; 9: 707-715.

32 Fan SH. Sharp asymmetric line shapes in side-coupled waveguide-cavity systems. Appl Phys Lett 2002; 80: 908-910.

33 Francardi M, Balet L, Gerardino A, Chauvin N, Bitauld D et al. Enhanced spontaneous emission in a photonic-crystal light-emitting diode. Appl Phys Lett 2008; 93: 143102 .

34 Intonti F, Vignolini S, Riboli F, Vinattieri A, Wiersma DS et al. Spectral tuning and near-field imaging of photonic crystal microcavities. Phys Rev $B$ 2008; 78: 041401(R).

35 Verellen N, Sonnefraud Y, Sobhani H, Hao F, Moshchalkov VV et al. Fano resonances in individual coherent plasmonic nanocavities. Nano Lett 2009; 9: 1663.

36 Liu M, Lee TW, Gray SK, Guyot-Sionnest P, Pelton M. Excitation of dark plasmons in metal nanoparticles by a localized emitter. Phys Rev Lett 2009; 102: 107401.
37 Bao W, Melli M, Caselli N, Riboli F, Wiersma DS et al. Mapping local charge recombination heterogeneity by multidimensional nanospectroscopic imaging. Science 2012; 338: 1317

38 Vignolini S, Intonti F, Riboli F, Wiersma DS, Balet L et al. Polarization-sensitive nearfield investigation of photonic crystal microcavities. App/ Phys Lett 2009; 94 : 163102

(c) (i) $\odot$ This work is licensed under a Creative Commons Attribution-

NonCommercial-NoDerivs 4.0 Unported License. The images or other third party material in this article are included in the article's Creative Commons license, unless indicated otherwise in the credit line; if the material is not included under the Creative

Commons license, users will need to obtain permission from the license holder to reproduce the material. To view a copy of this license, visit http://creativecommons.org/licenses/ by-nc-nd/4.0/ 